\title{
Propuesta para el tratamiento de problemas de tasas de variación relacionadas mediante el uso de GeoGebra: Un estudio de casos
}

\author{
Wendolyn E. Aguilar-Salinas*, Maximiliano De Las Fuentes-Lara, Araceli C. Justo-López y Ana D. Martínez-Molina \\ Facultad de Ingeniería, Universidad Autónoma de Baja California, Blvd. Benito Juárez S/N Col. Ex-ejido Coahuila, \\ Mexicali, México. Correo-e: aguilar.wendolyn@uabc.edu.mx; maximilianofuentes@uabc.edu.mx; \\ araceli.justo@uabc.edu.mx; ana.dolores.martinez.molina@uabc.edu.mx \\ * Autor a quien debe ser dirigida la correspondencia.
}

Recibido Mar. 1, 2021; Aceptado May. 4, 2021; Versión final Jun. 25, 2021, Publicado Oct. 2021

\begin{abstract}
Resumen
El principal objetivo de esta investigación es evaluar una estrategia educativa diseñada expresamente como plan de acción para el tema de tasas de variación relacionadas de la unidad de aprendizaje de cálculo diferencial durante la pandemia COVID-19. La metodología se basa en un paradigma cualitativo con la epistemología constructivista y en un enfoque semiótico en la enseñanza y aprendizaje de las matemáticas. Se utilizaron videoconferencias, secuencias didácticas y un problemario. Los resultados muestran que existe una mejora significativa de los estudiantes en relación al lenguaje matemático y a las aplicaciones conceptuales del cálculo diferencial, principalmente en los conceptos de tasas de variación y en la manipulación algebraica, así como en la transferencia de registros semióticos, independizando el concepto de sus representaciones. Se concluye que la enseñanza y aprendizaje utilizando estrategias didácticas apoyadas con GeoGebra, permite a los estudiantes investigar y mejorar su capacidad de abstracción, pensamiento lateral y persistencia mediante la integración de habilidades de pensamiento y comprensión conceptual.
\end{abstract}

\section{Proposal for the treatment of problems with related rates of variation through the use of GeoGebra: a case study}

\begin{abstract}
The main objective of this research study is to evaluate an educational strategy specifically designed as an action plan for the topic of related variation rates in the differential calculus on-learning unit during the COVID19 pandemic. The methodology is based on a qualitative paradigm with constructivist epistemology and on a semiotic focus on teaching and learning mathematics. Videoconferences, teaching sequences, and problems are used. The results show a significant improvement of students in their abilities related to mathematical language and conceptual applications of differential calculus, mainly in the concepts of variation rates and algebraic manipulation. Improvements are also observed in the transfer of semiotic records, independent of the concept of their representations. It is concluded that teaching and learning by using didactic strategies supported with GeoGebra, allow students to conduct research and improve their abstract capacity, their lateral thinking, and their persistence by integrating thinking abilities and conceptual comprehension.
\end{abstract}

Keywords: differential calculation; Duval's theories; mathematics; teaching-learning process; didactic sequences 


\section{INTRODUCCIÓN}

Debido a la contingencia en materia de salud que trajo consigo el COVID19 se restringió el acceso a las escuelas públicas y privadas de todos los niveles educativos en México, se eliminó la presencia física del personal docente, administrativo y estudiantil, para evitar los contagios que podrían darse en las escuelas y en los salones de clase. Como consecuencia se dieron condiciones excepcionales y complicadas en el entorno educativo, dirigiendo los esfuerzos de los docentes a mantener el nivel de actividad planeado para sus clases, pero migrando de manera automática sus clases presenciales a clases en línea.

Estos cambios no resultaron específicos de un área en particular de la docencia, sino que afectaron a todos por igual. Es por esto que los maestros del área de las matemáticas se encontraron frente a nuevas exigencias didácticas cambiantes e innovadoras que requerían un mayor tiempo de investigación en el campo de la didáctica y sobre todo al desarrollo de las unidades de aprendizaje para el tratamiento de una gran variedad de temas dentro y fuera de esta área (Mora, 2003), aunado a las dificultades de comprensión por parte de los estudiantes a ciertos conceptos importantes en las matemáticas, ha propiciado que los docentes permanezcan en una búsqueda constante de la mejor estrategia didáctica que permita superarlas.

Hay que tomar en cuenta que estas nuevas generaciones de estudiantes han nacido en la era digital y que traen consigo un enfoque significativamente diferente del aprendizaje y que el uso de las Tecnologías de Información y Comunicación (TIC) son parte de ellos. Investigaciones recientes han encontrado que la utilización de las TIC aumenta la aprobación de los cursos, ya que aumenta la participación de los estudiantes en la clase y en los entornos virtuales, permitiendo la formación de espacios de aprendizaje cada vez más interactivos que involucran conocimientos, experiencias y diversas prácticas entre docentes y estudiantes, gracias a los recursos tecnológicos educativos que poseen (Montenegro, 2016).

Dentro de la Universidad Autónoma de Baja California (UABC), el Centro de Educación Abierta y a Distancia (CEAD), es la instancia responsable de proporcionar los servicios institucionales de administración para los cursos en línea, ya sea en la modalidad semipresencial o a distancia, los cuales iniciaron en el semestre 20122 e incluyeron actividades asociadas a la formación docente, diseño instruccional (DI) y métodos de evaluación (Avitia et al., 2014). La unidad de aprendizaje (UA) de Cálculo Diferencial no fue ajena a este proceso implementándose de manera semipresencial. En el transcurso de los ciclos escolares 2016-2 a 20192 para esta UA no se encontró diferencias significativas en las evaluaciones de los estudiantes según la modalidad de aprendizaje. Por lo que una vez indicado el cese de labores el 18 de marzo del 2020, la Facultad de Ingeniería Mexicali (FIM) indico a sus docentes continuar las clases en la modalidad virtual hasta que las condiciones sanitarias se tornaran apropiadas para el regreso a clases presenciales.

Existe una abundante cantidad de estrategias didácticas basadas en recursos tecnológicos, como videos, applets o interactivos, destinados a facilitar la enseñanza y el aprendizaje de las matemáticas, pero para que estos recursos educativos desempeñen un buen papel en el aprendizaje, deben incitar la actividad y la reflexión matemática. Las estrategias didácticas tienen como propósito promover el desarrollo constructivista y significado de los conceptos, se trata de que los estudiantes utilicen un estilo heurístico para descubrir o redescubrir conocimiento, al aprovechar las tecnologías como herramientas que ayudan a la exploración, conjeturación y comprobación de las ideas matemáticas. El diseño e implementación de estas estrategias didácticas en el aula proporciona a los estudiantes una enseñanza basada en la experimentación, visualización y aplicación de los conceptos estudiados (Molon y Sidney, 2015). Actualmente, la computadora y los programas de cómputo se han convertido en el medio digital más difundido para el tratamiento de diferentes temas matemáticos que van desde juegos y actividades básicas hasta teorías y conceptos altamente complejos en el campo de las aplicaciones, ayudando al docente para un buen desempeño en el desarrollo del proceso de enseñanza y aprendizaje (Mora, 2003).

Como parte de la estrategia didáctica implementada en la UABC, se utiliza GeoGebra por ser un programa de aplicación de aprendizaje de matemáticas que soporta una gran diversidad de temas y está disponible libremente. Este software, tiene como objetivo mejorar la enseñanza y el aprendizaje de las matemáticas para todos los niveles educativos, combina formas dinámicas e interactivas en áreas como geometría, álgebra, cálculo, análisis y estadística. Además de que proporciona una buena base matemática y permite crear redes de conocimiento colaborativo que mejora el proceso de aprendizaje (Arango et al., 2014), por lo que contribuye su uso generalizado en la resolución de problemas matemáticos cotidianos. El uso del software GeoGebra es beneficioso en los salones de clase, porque constituye una herramienta de trabajo que capta la atención de los estudiantes, haciéndolos más participativos, creativos y proactivos, mejorando el proceso de enseñanza y aprendizaje (Rojas et al., 2019), además de permitir a los estudiantes adquirir conceptos matemáticos y habilidades en la resolución lógica y sistemática a través del proceso de descubrimiento y experiencia (MdAli y Kim, 2018). 
La resolución de problemas particularmente los de tasas de variación, que para la FIM se encuentra en la última unidad de la carta descriptiva de la UA de Cálculo Diferencial, representan una vía para atender algunas de las dificultades que los estudiantes presentan en el establecimiento de conexiones entre aquellos conceptos de fuertes componentes dinámicos, sus representaciones gráficas y el movimiento real de los objetos en sus contextos. Investigaciones han encontrado que estas dificultades se presentan principalmente por procedimientos basados en manipulaciones algebraicas descuidando los aspectos conceptuales y las relaciones con otras áreas del conocimiento (Areaya y Sidelil, 2012; Orhun, 2012), así como a una comprensión deficiente de funciones que no permite la modelización, derivación y la relación de las mismas cuando una es dependiente de la otra (Areaya y Sidelil, 2012; Berry y Nyman, 2003).

Durante el ciclo escolar 2019-2 se aplicó un curso remedial denominado Funciones y Derivadas para solventar las deficiencias de los estudiantes en tópicos del Cálculo Diferencial, con una participación de 300 estudiantes inscritos en la FIM, en el cual se incluyeron materiales didácticos, enlaces web y sugerencias de la resolución de ejercicios y problemas, así como el apoyo de un instructor en línea para solventar las dudas de los estudiantes. Con el propósito de evaluar el avance de los estudiantes, se diseñaron sondeos para cada uno de los temas del curso remedial. En el sondeo correspondiente al tópico de problemas de tasas de variación, los resultados permiten observar que solo el $51 \%$ de los estudiantes resolvió los problemas de variación de manera correcta, el resto evidenció las siguientes dificultades: no identificaron correctamente las variables dependientes, no identificaron correctamente las condiciones iniciales del problema, no declararon exactamente lo que se solicitaba en el problema, elaboraron las derivadas implícitas de manera incorrecta, no transitaron de manera correcta del lenguaje natural al lenguaje algebraico y los esquemas representativos de la situación fueron deficientes. Este sondeo sirvió de pre-test y permitió identificar aspectos a mejorar en el diseño e implementación de estrategias de intervención pedagógica a favor del desarrollo de competencias relacionadas con el aspecto algebraico y geométrico propio en la elaboración de funciones matemáticas (Alvarado et al., 2019).

Para que esta estrategia didáctica funcione se debe fortalecer en los estudiantes las habilidades necesarias que permitan desarrollar una concepción estructural de los conceptos asociados al Cálculo Diferencial, dentro de las cuales se encuentran: el razonamiento covariacional, el concepto de función y las relaciones de proporcionalidad (Mercapide, 2018), así como las habilidades matemáticas propuestas por Krutetskii (1976): analítica, geométrica y armónica, dentro de las cuales el estudiante tiene que lograr el dominio del componente lógico-verbal, del componente pictórico-visual y el equilibrio entre los dos componentes.Para determinar las habilidades adquiridas por los estudiantes después de implementada la estrategia, se evaluó el rendimiento, por medio de la observación indirecta de la actuación de los estudiantes en situaciones problemáticas reales o simuladas ya que por el distanciamiento social no se podía realizar la observación directa, y se evaluaron las habilidades reales de resolución de problemas y el rendimiento, por medio de pruebas escritas de solución de problemas (reales o hipotéticos), y autorregistros (D’Zurilla y Nezu, 2007).

\section{OTROS ANTECEDENTES}

Se abordará la epistemología constructivista como base orientadora del proceso de enseñanza-aprendizaje, entendiendo que el ser humano es activo constructor de su realidad, pero siempre en interacción con otros. Desde el constructivismo se puede decir que el proceso de enseñanza-aprendizaje es una interacción dialéctica entre los conocimientos del docente y los de los estudiantes, que entran en discusión, oposición y diálogo, para llevar una síntesis productiva y significativa: el aprendizaje; y que está determinado por un contexto específico debido a las condiciones biológicas, psicológicas, sociales, económicas, culturales, entre otras, de cada uno (Ortiz, 2015). Para determinar cómo se dan estos aprendizajes, se consideran las teorías de aprendizaje de Piaget, Ausubel y Vygotsky por estar más acordes a los planteamientos del constructivismo.

La teoría cognoscitiva de Piaget establece que el aprendizaje es un proceso que sólo tiene sentido ante situaciones de cambio, por lo que el aprendizaje es concebido como la adaptación a esos cambios, mediante dos procesos esenciales: la asimilación y la acomodación. Donde la asimilación hace referencia a la manera en que un organismo enfrenta un estímulo externo en base a sus leyes de organización presentes y la acomodación, por el contrario, involucra una modificación en la organización presente en respuesta a las exigencias del medio (Saldarriaga-Zambrano et al., 2016). La teoría del aprendizaje significativo de Ausubel menciona que los estudiantes no comienzan su aprendizaje desde cero, sino que aportan a este proceso sus experiencias y conocimientos, condicionando aquello que aprenden, los cuales si son conducidos y manipulados adecuadamente pueden ser aprovechados para mejorar el proceso de aprendizaje y para hacerlo significativo (Ortíz, 2015).

La teoría del aprendizaje social de Vygotsky sostiene que el aprendizaje es el resultado de la interacción del individuo con el medio. En esta teoría es importante lo que Vygotsky ha denominado la zona de desarrollo próximo, que es la distancia entre lo que una persona puede aprender por sí misma y lo que podría aprender con la ayuda de un experto en el tema, produciendo el aprendizaje de nuevas habilidades que el ser humano 
pone a prueba en distintos contextos (Ortiz, 2015). Dentro de esta interacción con otros que mencionan las teorías constructivistas para la adquisición de aprendizajes, el método heurístico permitirá al estudiante a través de una serie de pasos y procedimientos de aprendizaje, seleccionar el momento adecuado para retroalimentar los contenidos que hayan sido adquiridos de manera deficiente por parte del docente, el cual actuará como guía o tutor, planteando problemas, suministrando material, fomentando la responsabilidad e iniciativa del estudiante, conduciendo al estudiante a la búsqueda del conocimiento, estimulando su capacidad de reflexión, guiándolo para que indague, investigue y llegue a sus propias conclusiones (Fortea, 2003).

Desde el punto de vista constructivista una experiencia que favorece el aprendizaje de las matemáticas y que favorece la construcción de conocimientos a partir de procesos abstractos reflexiva es la resolución de problemas. Para Polya (1965) la resolución de problemas consiste en cuatro fases bien definidas: la comprensión del problema, la concepción de un plan, la ejecución del plan y una visión retrospectiva. Considerando que para que el estudiante construya su propio conocimiento y lleve a cabo la interacción activa con los objetos matemáticos (OM), es indispensable la reflexión que le permita abstraerlos, encontrarlos inmersos en un problema y no en un ejercicio, para producir un desequilibrio en las estructuras mentales del estudiante, que en su afán de equilibrarla construya el conocimiento.

El enfoque semiótico en la enseñanza y aprendizaje de las matemáticas nació a raíz de las dificultades sobre la comprensión y la necesidad de recurrir a otros tipos de representaciones que constituyen el lenguaje de las matemáticas. Según la teoría de representaciones semióticas de Duval (2006) hay por lo menos dos características de la actividad cognitiva implicadas en las estrategias matemáticas: varios registros de representación semiótica y los OM. Dado que una estrategia matemática combina generalmente tratamientos y conversiones, la diferenciación funcional de registros de representación y la coordinación entre ellos constituyen los dos puntos clave para el aprendizaje.

Desde la perspectiva de la Teoría de Representaciones Semióticas (TRS), los OM no son directamente accesibles a la percepción, consecuentemente para su estudio y tratamiento se requiere contar con representaciones de los mismos, las representaciones externas a las que se hace alusión pueden ser de carácter geométrico, algebraico y numérico. Como lo señala el mismo Duval (2000), el uso de sistemas de representaciones semióticas para el pensamiento matemático es esencial, debido a que a diferencia de otros campos de conocimiento (biología, geología, física), no existen otras maneras de lograr el acceso a los OM sino a través de representaciones. Estos procesos de representación, tratamiento y conversión permiten exteriorizar las representaciones mentales de los individuos, motivando la retroalimentación y mejoramiento de las mismas, que conduce a la formación de preceptos matemáticos y a la conceptualización del OM. La $\mathrm{AC}$ de representación constituye una marca o conjunto de marcas perceptibles e identificables respecto de un $\mathrm{OM}$, el tratamiento es la transformación de la representación dentro del mismo registro de representación, y la conversión es la transformación de la representación en otra representación de un registro diferente al original pero que conserva su esencia (Duval, 2006).

El objetivo de esta investigación es evaluar el impacto de una estrategia didáctica que se diseñó para llevarse a cabo durante el distanciamiento social producido por la pandemia del COVID19. El propósito de la estrategia didáctica diseñada es que el estudiante alcance las habilidades y competencias para la resolución de enunciados de problemas de tasas de variación relacionadas de la unidad de aprendizaje de Cálculo Diferencial en modalidad virtual. Para desarrollar esta habilidades y competencias, el estudiante deberá definir las variables de la ecuación que obtendrá, escribir los hechos numéricos conocidos acerca de las variables y sus derivadas con respecto al tiempo, declarar lo que se desea calcular, escribir una ecuación que relacione las variables, derivar e interpretar los resultados.

Mediante la estrategia didáctica el estudiante navegará a través de las distintas teorías de aprendizaje, al manipular las simulaciones o programas interactivos y emplear distintos valores en las variables del problema para observar los cambios que se producen en el fenómeno planteado, el estudiante transitará por la teoría cognoscitiva de Piaget, donde asimilará los cambios producidos y se adaptará al cambio. Al desarrollar o modelar el problema matemáticamente, el estudiante hará gala de sus conocimientos previos adquiridos en las etapas educativas anteriores como lo menciona la teoría de aprendizaje significativo de Ausubel, al resolverlo utilizará los cuatro pasos de la teoría de Polya para la resolución de problemas y las teorías cognitivas de Duval para los cambios en las representaciones semióticas de los OM, siempre apoyado del docente y sus compañeros mediante la comunicación directa como lo indica Vygotsky en la zona de desarrollo próximo.

\section{MÉTODO}

En la investigación se optó por el paradigma cualitativo y por una metodología de investigación-acción, vinculado a la puesta en práctica con la estrategia didáctica en un estudio de casos. Toda vez que se pretende entender el fenómeno de estudio. 


\section{Sujetos}

En esta investigación participaron seis alumnos inscritos en la unidad de aprendizaje de Cálculo Diferencial en el ciclo escolar 2020-1 de la Facultad de Ingeniería Mexicali de la Universidad Autónoma de Baja California, México.

\section{Instrumentos}

Para lograr el propósito de la investigación se desarrolló una estrategia didáctica que incluía: videoconferencias, secuencias didácticas y un problemario. La primera parte de la estrategia didáctica se desarrolló mediante dos videoconferencias utilizando la plataforma Blackboard Collaborate. Una videoconferencia estaba enfocada a los conceptos de derivación implícita, derivadas con respecto al tiempo y tasas de variación; y la otra en la resolución de ejemplos de tasas de variación relacionadas propuestos por el docente.

La estrategia didáctica se diseñó a partir de las teorías cognitivas de Duval toda vez que en las actividades los estudiantes tienen que articular de un registro de representación (algebraico, numérico y geométrico) a otro y los avances logrados con la incorporación de la tecnología, particularmente con el software GeoGebra (Arango et al., 2015; Caligaris et al., 2015). La estrategia didáctica utilizada se considera instruccional de acuerdo a Feo (2010) toda vez que se pretende que el estudiante logre objetivos particulares, la interrelación entre el docente y estudiante es indispensable, se basa en hojas de trabajo e incluye recurso tecnológico como mediador entre el sujeto y el objeto de conocimiento.

Esta estrategia didáctica incluyó programas interactivos y hojas de trabajo. Los programas interactivos como páginas web fueron especialmente diseñados para representar ejemplos de problemas de tasas de variación relacionadas, como: el caso de la bacteria, el caso del abrevadero de forma trapezoidal y el caso del avión que vuela con rapidez constante. Estos representan una alternativa grafica de visualización como un complemento de las representaciones habituales utilizadas por el docente en la clase. En estas simulaciones el estudiante puede modificar los parámetros del problema, observar los cambios en la representación gráfica y razonar sobre los mismos en la representación algebraica, reflexionando sobre los cambios y lo que estos significan en la situación problémica. Adicionalmente va llenando las hojas de trabajo que lo van guiando en el descubrimiento de nuevo conocimiento. En la figura 1 se muestra a manera de ejemplo el problema del abrevadero de forma trapezoidal, el cual permite al estudiante modificar el largo del tanque y el flujo del agua.

\section{Problema del Trapecio}

Un depósito horizontal para agua mide cierto largo en metros y sus extremos son trapecios con una altura de $4 \mathrm{~m}$, base menor de $4 \mathrm{~m}$ y base mayor de $8 \mathrm{~m}$. Se vierte agua en el depósito a una tasa de ciertos metros cúbicos por minuto. ¿Qué tan rápido sube el nivel h del agua cuando ésta ha alcanzado cierta profundidad?

\section{Datos de entrada}

Largo del tanque en metros

Flujo de agua en metro cúbico por

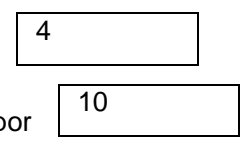
minuto

Botones de control

\begin{tabular}{|l|}
\hline Llenado del tanque \\
\hline \hline Abrir llave de agua \\
\hline
\end{tabular}
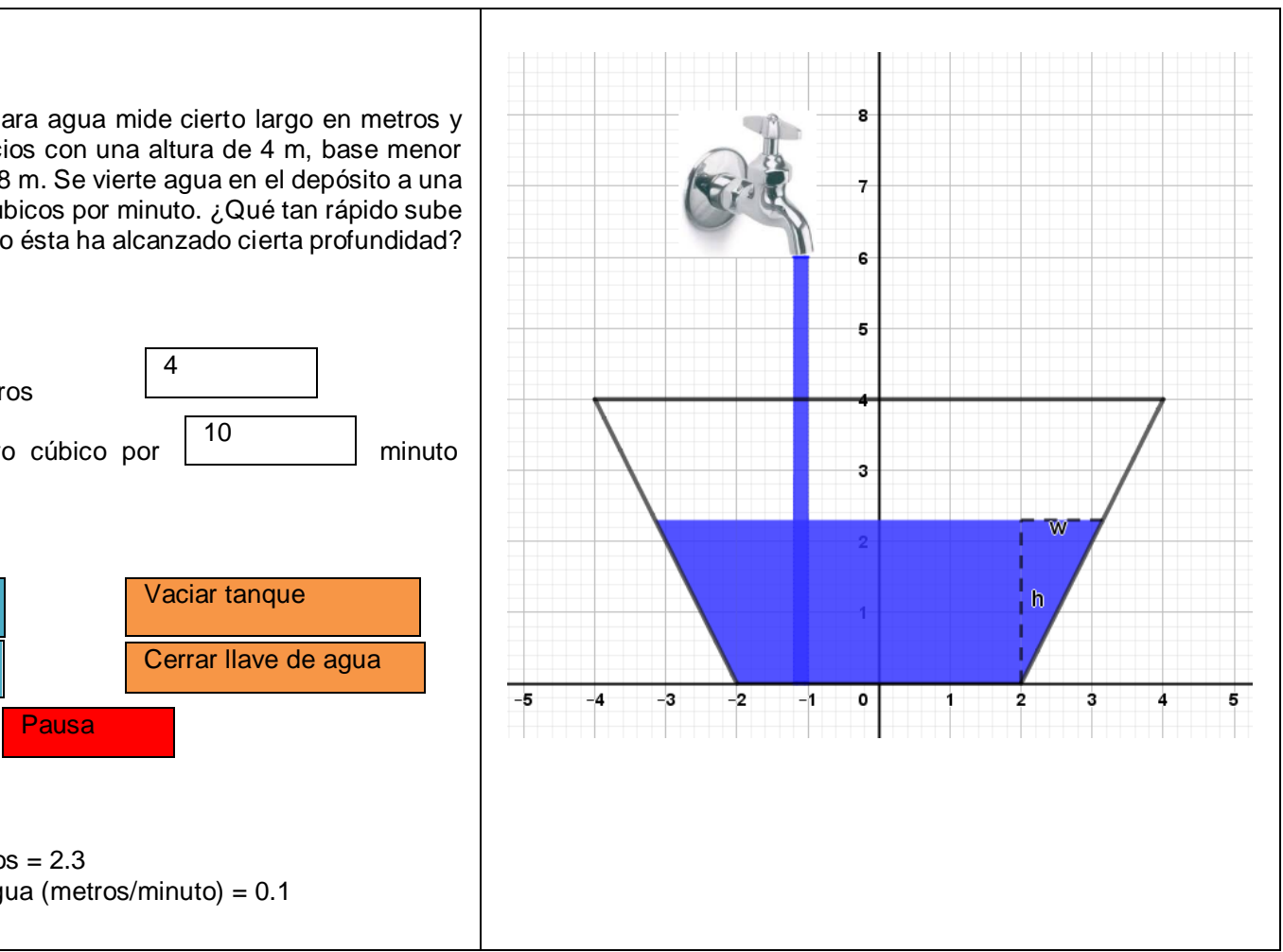

Cálculos

Nivel $\mathrm{h}$ de agua en metros $=2.3$

Variación del nivel del agua (metros $/$ minuto) $=0.1$

Fig. 1: Problema de tasa de variación: el caso del abrevadero de forma trapezoidal 
Los programas interactivos fueron diseñados como complemento de las hojas de trabajo, con el propósito de guiar a los estudiantes mediante preguntas elaboradas en el uso de la simulación, se enfocan principalmente en la resolución de enunciados de tasas de variación relacionadas que tienen una función implícita como modelo matemático, en la adquisición de los conceptos del cálculo y no en la reproducción o mecanización de procedimientos establecidos. El problemario consta de 10 problemas de tasas de variación relacionadas recopilados del libro de Cálculo I (Larson et al., 2010), para su resolución el alumno se basa en la explicación del docente mediante videoconferencia, las hojas de trabajo y los programas interactivos. El docente permanece como guía atendiendo dudas sobre la resolución de cada ejercicio vía correo electrónico o foro de discusión de la plataforma Blackboard. Entre los problemas a resolver solicitados se encuentran: el abrevadero de forma triangular, la bacteria celular de forma esférica, los dos automóviles que se aproximan a un crucero, entre otros.

\section{Método de evaluación}

Como parte final se diseñó una prueba que permitiera indagar sobre los conocimientos y habilidades adquiridas por los estudiantes. Dicha prueba se diseñó con 16 reactivos de opción múltiple, cinco derivadas de funciones algebraicas, cinco derivadas de funciones trascendentes, dos derivaciones implícitas, una derivada por definición, una derivada con respecto al tiempo y dos problemas de tasas de variación relacionadas que el estudiante tenía que modelar y resolver. Adicionalmente, el estudiante tenía que subir los procedimientos que lo hicieran llegar a esa solución. Los problemas del examen no formaban parte del problemario o de las hojas de trabajo.

La validez de contenido de la prueba se garantizó con la participación de jueces expertos (profesores de cálculo diferencial con experiencia docente mínima de 5 años) y método de consenso grupal, en los temas objeto de la validación quienes analizaron la coherencia de los reactivos con lo que se desea evaluar, la complejidad de los reactivos y la habilidad cognitiva a evaluar, así como la suficiencia y pertinencia de los reactivos, considerando los aspectos del constructo que son relevantes, incluidos en las competencias y los indicadores (Cisneros et al., 2012).

\section{RESULTADOS Y DISCUSIÓN}

Debido a las circunstancias que el COVID19 ha puesto a la educación en México, fue necesaria la utilización de nuevas estrategias en todos los niveles educativos y en la educación superior no fue la diferencia. Los docentes de la Facultad de Ingeniería de la Universidad Autónoma de Baja California han tenido que mejorar sus estrategias de enseñanza enfocándolas principalmente en el aprendizaje en línea. En esta medida, se destaca el rol del docente como aquel que debe asumir nuevos enfoques en sus prácticas educativas, partiendo de una planificación dirigida al uso de nuevas metodologías y estrategias de enseñanza que promuevan el autoaprendizaje, la transferencia de conocimientos a situaciones reales y la evaluación de la estrategia para el logro de los objetivos (Montenegro, 2016).

Para el cumplimiento de la primera parte de la estrategia se desarrollaron dos videoconferencias en la plataforma Blackboard Collaborate en las que el docente plantea los antecedentes y conceptos relacionados al tema de variación, así como en la resolución de problemas de aplicación. Sin embargo, se pudo observar que solo el $58 \%$ de los estudiantes se encontraban en tiempo para la explicación, aumentando la dificultad de los alumnos a comprender el tema sin la explicación previa del docente. La baja participación demostró en muchos casos: la falta de conectividad a internet, la ausencia de equipo de cómputo o dispositivo móvil y en algunos casos la falta de responsabilidad del estudiante con la clase.

Dentro de las principales ventajas de utilizar la plataforma Blackboard Collaborate se encontraron las siguientes: permite un contacto sincrónico y bidireccional con los estudiantes, posibilita distintas actividades y la grabación de la sesión, permite complementar y/o enriquecer el contenido de la asignatura, facilita la comprensión de conceptos a través de una retroalimentación inmediata, tiene la opción de compartir el escritorio, por lo que se puede visitar páginas web, mostrar aplicaciones y compartir archivos (Villalón et al., 2019), propiciando herramientas y procesos para la interacción comunicativa dirigida al aprendizaje (Montenegro, 2016). Tomando como base sus ventajas, la estrategia radicó en hacer grabaciones de las sesiones realizadas con los estudiantes que podían ser compartidas y descargada posterior a la sesión para ser estudiadas y analizadas nuevamente.

Las hojas de trabajo guían a los estudiantes en el uso de los programas interactivos para adquirir el conocimiento y las habilidades deseadas por el docente. Primeramente, el estudiante tuvo que leer las hojas de trabajo para posicionarse en una situación problémica, poner en marcha el interactivo y observar lo que sucedía, para posteriormente responder las preguntas de las hojas de trabajo relacionadas al tema. Al manipular los programas interactivos, emplear diferentes valores en las variables del problema y observar los cambios que se producían en éste, el estudiante recae en la teoría cognoscitiva de Piaget donde el aprendizaje está basado en un proceso de adaptación de cambios, que por medio de la asimilación y la 
adaptación construye su aprendizaje. GeoGebra puede fomentar la resolución activa de problemas centrados en los estudiantes al permitir experimentos matemáticos, exploraciones interactivas, así como la enseñanza de descubrimiento (Prodromou, 2015).

Para el caso del problema del abrevadero de forma trapezoidal, respondió preguntas como: conforme el flujo de agua cae al abrevadero ¿qué está variando?, ¿cuál es la variable independiente?, ¿cuál es el valor de w en el momento en que $h=1$ metro?, determina una función con la que puedas calcular $w$ en términos de la variable $h$, ¿cuál es el valor del área húmeda $(A)$ en el momento en que $h=1$ metro?, determina una función con la que puedas calcular $A$ en términos solo de la variable $h$, es decir $A(h)$, entre otras. Dentro del contexto del problema, se le pide al estudiante buscar entre el conocimiento anteriormente adquirido como menciona la teoría de aprendizaje significativo de Ausubel incorporando al proceso sus propias experiencias y conocimientos, para encontrar la manera de relacionar el volumen del tanque en forma trapezoidal y la variación del nivel de profundidad del agua con respecto al tiempo y buscar la solución al problema (Figura 2).

1. Conforme el flujo de agua cae en el abrevadero. ¿Qué está variando? Define esas variables

$$
\begin{array}{ll}
h=\text { altura del agua (dependiente) } & w=\text { ancho del tanque (dependiente) } \\
t=\text { tiempo (independiente) } & v=\text { volumen del agua (dependiente) }
\end{array}
$$

2. ¿Cuál es la variable independiente?

Tiempo

3. ¿Cuál es el valor de $w$ en el momento en que $h=1$ metro? Determina una función con la puedas calcular $w$ en términos de la variable $h$.

$$
w=\frac{1}{2} h \quad w=\frac{1}{2}(1) \quad w=0.5
$$

4. ¿Cuál es el valor del área húmeda $(A)$ en el momento en que $h=1$ metro? Determina una función con la que puedas calcular (A) en términos solo de la variable $h$.

$$
A(h)=4 h+\frac{h^{2}}{2} \quad w=\frac{1}{2} h \quad A h=4.5 m^{2}
$$

5. ¿Cuál es el volumen de agua $(V)$ en el tanque en el momento en que $h=1$ metro? Determina una función con la que puedas calcular $V$ en términos de la variable $h$, es decir $V(h)$.

$$
V(h)=\left(4 h+\frac{h^{2}}{2}\right) 4 \quad V(h)=\left(4(1)+\frac{(1)^{2}}{2}\right) 4 \quad V(h)=18 m^{3}
$$

6. De la expresión algebraica de volumen que obtuviste determina su variación con respecto al tiempo y calcula la variación del nivel del agua $h$ en el momento en que $h=2$ metros.

$$
\begin{gathered}
V=2 h^{2}+16 h \\
V^{\prime}=4 h h^{\prime}+16 h^{\prime} \\
V^{\prime}=h^{\prime}(16+4 h) \\
h^{\prime}=\frac{V^{\prime}}{16+4 h}
\end{gathered}
$$

$$
\begin{gathered}
h^{\prime}=\frac{10}{16+8} \\
h^{\prime}=\frac{5}{12} \mathrm{~m} / \mathrm{min}
\end{gathered}
$$

Fig. 2: Respuesta de un estudiante para solucionar el problema del abrevadero de forma trapezoidal

A partir de la puesta en escena de la simulación del abrevadero en forma trapezoidal el estudiante tiene la posibilidad de identificar las variables dependientes $\mathrm{h}$ (altura del nivel de agua), w (ancho del triángulo formado) y $v$ (volumen de agua en el tanque), en este caso transita de una representación gráfica a una algebraica, los conocimientos previos sobre triángulos semejantes aunado a la representación gráfica producida por la simulación favorece la producción de una función que relacione h y w, aunque el trabajo posterior del estudiante a partir del establecimiento de esta relación es predominantemente algebraico (en el que define y deriva una función implícita para obtener la variación del nivel de agua) y finalmente numérico, el origen de tal manipulación es una representación gráfica y está asociada a un contexto en lenguaje natural. La actividad y la simulación diseñada promueven y favorecen el tratamiento y la conversión de los registros de representación, punto clave para el aprendizaje (Duval, 2006).

Al incluir GeoGebra a la propuesta se busca que el estudiante construya los conceptos matemáticos a partir de la vinculación de los distintos registros de representación, así como también genere conocimiento a partir de su interacción con los objetos matemáticos, por lo que la teoría de representaciones semióticas juega un papel primordial a partir de las actividades cognitivas de tratamiento y conversión. A través de la manipulación, exploración y experimentación el estudiante extrae sus propias conjeturas, ideas y conclusiones, logrando un 
aprendizaje más duradero y significativo (Olivares y Valero, 2019), ya que mejora el conocimiento de los estudiantes, la capacidad de visualización espacial y el pensamiento crítico, creativo e innovador (Kim y MdAli, 2017). GeoGebra representa una herramienta útil para solventar las desventajas típicas de los estudiantes en el aprendizaje matemático, como la dificultad de visualizar las gráficas de la función o la falta de capacidad para seguir los pasos algebraicos sin cometer errores en el cálculo (Criscuolo y Gnudi, 2013).

La revisión de lo plasmado por los estudiantes en las hojas de trabajo permitió observar que la secuencia didáctica conduce a una mejora significativa de los estudiantes, en relación al lenguaje matemático y a las aplicaciones conceptuales en el Cálculo Diferencial. Los estudiantes identificaron y manipularon el movimiento de las variables, adquiriendo recursos propios para realizar transferencias de registros semióticos e independizaron el concepto de sus representaciones (Báez et al., 2018), ayudando a superar las dificultades en el análisis de conceptos abstractos, especialmente en el análisis de gráficos e imágenes (Hartini et al., 2020). Parte del objetivo de la estrategia didáctica es que los estudiantes trabajen con distintos tipos de representaciones y que sean vistas como objetos susceptibles a ser transformados.

La aprehensión conceptual limitada de los estudiantes a los OM debido en parte al énfasis en la praxis algorítmica e insuficiente trabajo de conversión de registros de representación durante las clases de Cálculo Diferencial, por lo que la actividad cognitiva de conversión evidencia la mayor dificultad para los estudiantes cuando esta se presenta como necesaria para la resolución de un problema. Por lo que la resolución de problemas constituye una herramienta didáctica que permite desarrollar habilidades en los estudiantes, además de ser una herramienta de fácil transferencia para la vida, puesto que permite al estudiante enfrentarse a situaciones y problemas que debe resolver.

Para el desarrollo del problemario, el estudiante tuvo que utilizar los cuatro pasos de Polya (1965) para la resolución de un problema: comprender el problema, concepción de un plan, ejecución del plan y examinar la solución obtenida, así como del foro de dudas en el cual guiado por el docente y con las sugerencias e ideas de los compañeros de clase se podía encontrar una solución al problema, como lo indica Vygotsky en la zona de desarrollo próximo. Para comprender el problema de los dos automóviles que se aproximan a un crucero (Figura 3), el estudiante debe contextualizar el problema, respondiendo preguntas como: ¿Cuáles son los datos?, ¿Cuáles son las condiciones iniciales del problema? ¿Cuál es la ecuación que puedes utilizar para relacionar las variables dependientes e independientes en el problema? Esta es la fase más complicada para los estudiantes ya que deben considerar alternativas posibles y viables para darle solución al problema considerando la naturaleza del mismo.

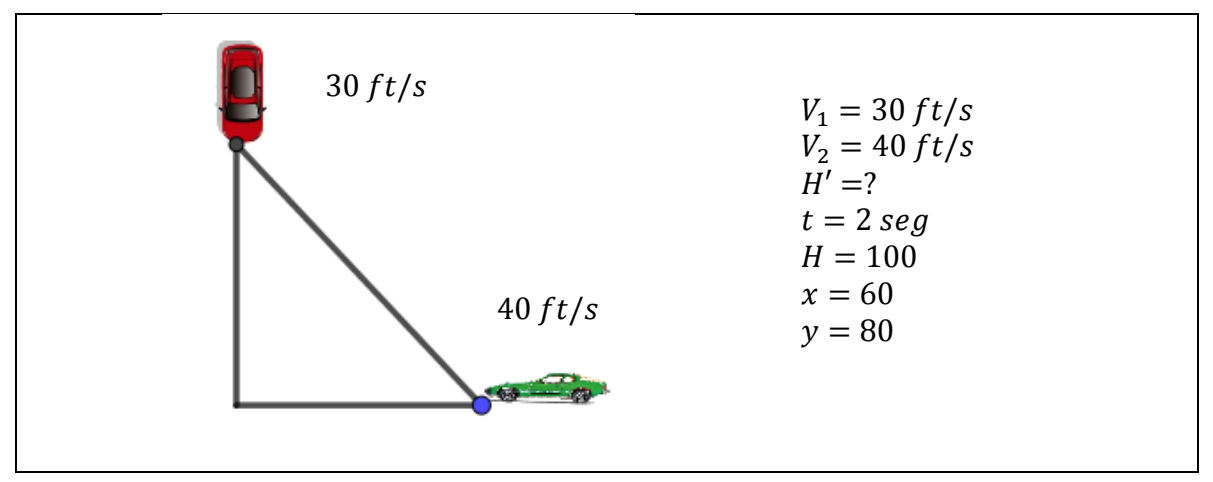

Fig. 3: Primer paso-comprender el problema

En la segunda fase, la concepción de un plan, el estudiante se encuentra en el preámbulo de utilizar una metodología (Figura 4). Para esto, Polya (1965) sugiere revisar problemas similares al que se está enfrentando, analizar procedimientos que alguien más ha realizado. Para el caso del ejemplo, el estudiante ha ideado utilizar el teorema de Pitágoras. En la fase tres, se desarrolla el plan de acción, en ésta el estudiante debe retroalimentar constantemente su andar hasta obtener resultados favorables que le permitan darle solución al problema (Figura 5). En el cuarto paso, examinar la solución obtenida en pies por segundo (Figura 6), el estudiante se enfrenta a su descubrimiento, el cual puede trascender como una nueva metodología y extender a otro problema.

$$
x^{2}+y^{2}=H^{2}
$$

Fig. 4: Segundo paso-concebir un plan 


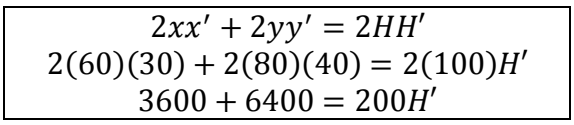

Fig. 5: Tercer paso-ejecutar el plan

$$
H^{\prime}=50 \mathrm{ft} / \mathrm{s}
$$

Fig. 6: Cuarto paso-examinar la solución obtenida

Otro ejemplo de la resolución de problemas por parte de los estudiantes puede observarse en la figura 7, con el problema del abrevadero con cara triangular. La resolución de problemas generalmente demuestra que los estudiantes son capaces de aplicar conocimientos y habilidades de razonamiento matemático en situaciones directas, por lo que resulta ser una parte indispensable de la educación matemática. Esta resolución de ejercicios permite verificar la frecuencia con la que los alumnos cometen errores de notación (incorrecta o insuficiente), errores de operación y simplificación, y errores de comprensión lectora que imposibilitan la correcta resolución de problemas (Mercapide, 2018).

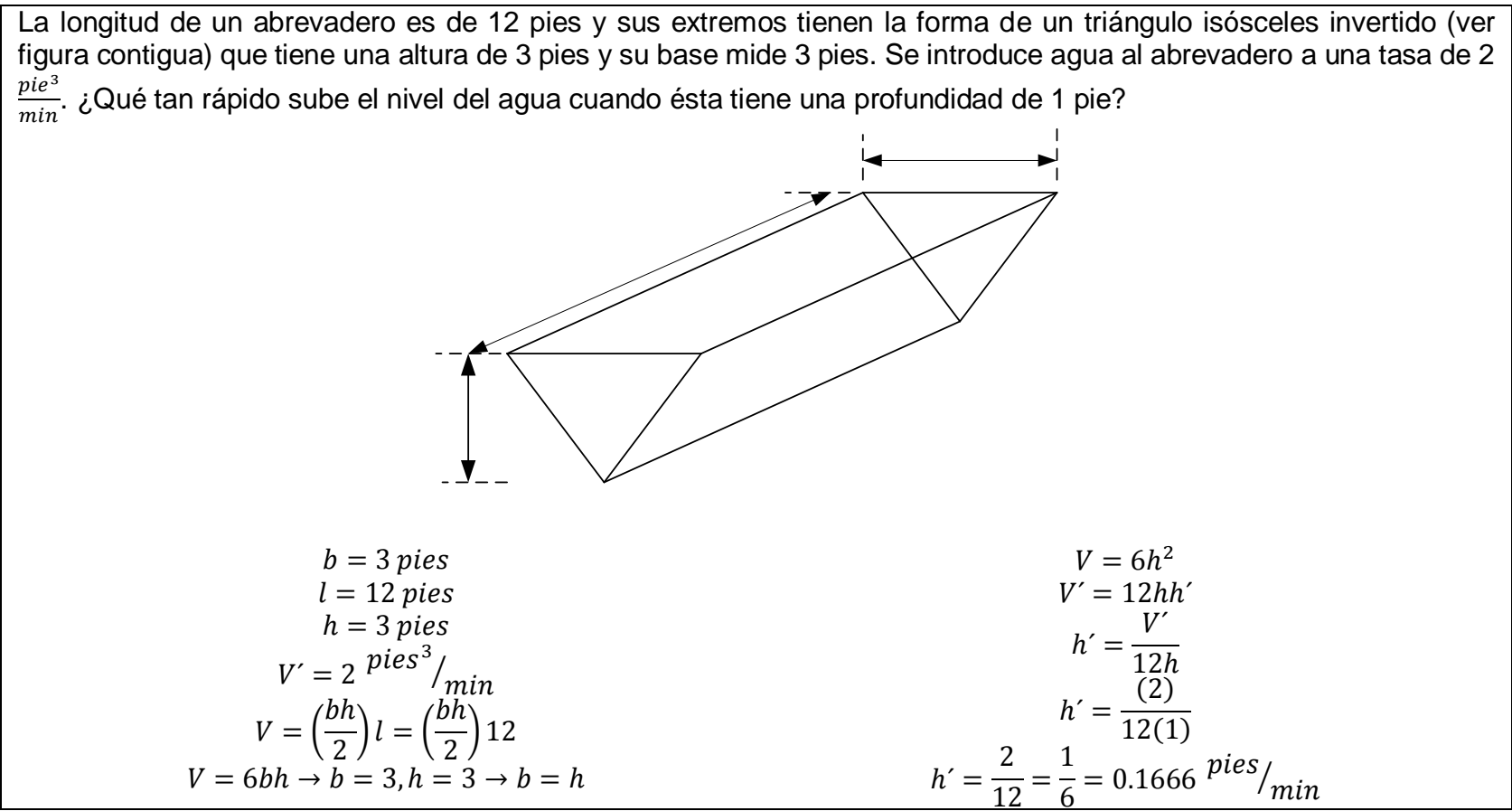

Fig. 7: Respuesta de un estudiante para solucionar el problema del abrevadero con cara triangular

Como parte de la evaluación final (post-test), se aplicó un examen el cual incluía dos problemas de tasas de variación relacionadas: el problema de la escalera y el problema del tanque en forma de cono. Entre las dificultades encontradas en los estudiantes que no utilizaron la estrategia didáctica se encontraron: la falta de comprensión al problema, el establecimiento incorrecto de los datos del problema, la omisión de constantes involucradas en el procesamiento algebraico, errores en el cálculo y procedimientos erróneos en el cálculo cuando se ven involucradas diferentes fórmulas de derivación. En algunos de los resultados de estos reactivos se puede observar que los estudiantes una vez realizado el procedimiento de manera correcta, se olvidan de sustituir la variable, las unidades en las que se plasma el contexto del problema o la conclusión del mismo (Figura 8).

\begin{tabular}{|c|c|}
\hline $\begin{array}{c}V=\frac{1}{3} \pi r^{2} h \\
\frac{r}{h}=\frac{4}{14}=\frac{2}{7} \\
r=\frac{2}{7} h \\
V=\frac{1}{3} \pi\left(\frac{2}{7} h\right)^{2} h \\
V=\frac{1}{3} \pi \frac{4}{49} h^{2} h\end{array}$ & $\begin{aligned} \frac{d V}{d t} & =\frac{12}{147} \pi h^{2} h^{\prime} \\
h^{\prime} & =\frac{147}{12 \pi h^{2}} \\
\text { Si } h=6 \text { entonces } & \\
h^{\prime} & =\frac{147}{432 \pi} \\
h^{\prime} & =\frac{49}{144 \pi}\end{aligned}$ \\
\hline
\end{tabular}

Fig. 8: Respuesta de un estudiante para solucionar el problema del tanque en forma de cono 
En cuanto a los estudiantes que utilizaron la estrategia didáctica, se observó que el $83 \%$ de los estudiantes resolvieron los problemas de tasas de variación de manera correcta. El estudiante que no logro completar estos problemas fue debido al desarrollo incorrecto de la derivación implícita de las funciones involucradas. El uso de nuevas alternativas pedagógicas permitió captar la atención y el interés de los estudiantes por aprender matemáticas y esto se reflejó en los resultados favorables después de la intervención al grupo experimental en el post-test (Alvarado et al., 2019). El uso de la tecnología ha demostrado mejorar el logro de los resultados de aprendizaje en las clases de matemáticas. Al razonar los estudiantes sobre un problema utilizando múltiples representaciones, experimentan el proceso de obtener su propia comprensión (Widjajanti et al., 2019).

\section{DISCUSIÓN FINAL}

La revisión de la estrategia didáctica permitió observar que la propuesta conduce a una mejora significativa de los estudiantes, en relación al lenguaje matemático y a las aplicaciones conceptuales del Cálculo Diferencial, principalmente en los conceptos de tasas de variación y en la manipulación algebraica, también los estudiantes realizaron transferencias de registros semióticos e independizaron el concepto de sus representaciones.

Con base en la Teoría de Representaciones Semióticas se diseñó e implementó una estrategia didáctica que incorpora hojas de trabajo, programas interactivos diseñados con el software GeoGebra y un problemario, con el propósito de mejorar los conocimientos y habilidades de los estudiantes en términos de las actividades cognitivas, las distintas representaciones semióticas y los conceptos asociados a las aplicaciones de tasas de variación relacionadas. La estrategia didáctica diseñada enfatiza en el uso de los diferentes registros de representación. Se incluye el software GeoGebra a la estrategia didáctica para que el estudiante construya los OM a partir de la vinculación de los distintos registros de representación, por lo que la Teoría de Representaciones Semióticas juega un papel primordial en la construcción de tales objetos.

Entre los errores más comunes que cometen los estudiantes, se observaron los siguientes: procedimientos basados en manipulaciones algebraicas descuidando los aspectos conceptuales y las relaciones con otras áreas del conocimiento, una comprensión deficiente de funciones que no permite la modelización, derivación y la relación de las mismas cuando una es dependiente de la otra, procedimientos mal ejecutados por el incorrecto uso del álgebra y la aritmética, deficiente comprensión lectora que imposibilitan la correcta resolución de problemas y un modelado adecuado, falta de comprensión al problema con el establecimiento incorrecto de los datos del problema, debido a inadecuadas relaciones de proporcionalidad, problemas en la resolución o procedimiento del problema, por la omisión de constantes involucradas en el procesamiento algebraico y errores en el cálculo, procedimientos erróneos en el cálculo cuando se ven involucradas diferentes reglas de derivación.

A pesar de las ventajas observadas en la enseñanza en línea, como: el favorecer el acceso a contenidos educativos y la comunicación entre profesores y estudiantes, el facilitar el intercambio de ideas, el aprendizaje cooperativo y al mismo tiempo permite la individualización del proceso de enseñanza-aprendizaje con actividades o tareas relevantes y creativas que el estudiante va realizando de manera autónoma controlando su ritmo y horario; se identificaron algunas desventajas que no son particulares de los problemas de tasas de variación, sino de la enseñanza a través de problemas, como lo son: el cambio entre registros semióticos particularmente entre el lenguaje natural al lenguaje algebraico y la identificación de los datos relevantes del problema.

El uso de las herramientas, comandos y funciones predefinidas que están disponibles en el software GeoGebra, permitió al docente elaborar materiales didácticos significativos, que pueden ser utilizados para la enseñanza y aprendizaje de los conceptos abordados y estimular la imaginación del estudiante. Es indispensable considerar que el contar con conocimientos sólidos en los estudiantes aumenta la probabilidad de mejorar en las materias de matemáticas subsecuentes, por lo que es necesario reflexionar sobre el modelo educativo que permite que los alumnos avancen en el sistema educativo. Se pretende posteriormente utilizar este conjunto de estrategias en grupos de control para comprobar cuantitativamente el éxito de la misma y utilizar la retroalimentación oportuna del docente. Se observó que el papel del docente es fundamental ya que despierta la creatividad y provoca en los estudiantes la capacidad para emocionarse en el aprendizaje.

\section{CONCLUSIONES}

De acuerdo al trabajo presentado y a los resultados obtenidos, se pueden plantear las siguientes conclusiones principales: 
1.- Se trabajó con una estrategia didáctica, que permitió lograr en el estudiante la generación de su propio conocimiento a través de un simulador o interactivo, donde podía observar los cambios en las representaciones semióticas del modelo de la situación problémica.

2.- La interacción directa con el docente a través de un foro de dudas, permitió que el estudiante se sintiera acompañado durante todo el trayecto de su aprendizaje.

3.- El reforzamiento del conocimiento a través de un problemario, consiguió en el estudiante poder transportar el conocimiento adquirido con la estrategia didáctica a otros tipos de situaciones.

4.- La estrategia didáctica planteada a través de las situaciones problémicas, promueve un acercamiento semiótico al lenguaje algebraico por parte de textos que permitan conjugar lo numérico y lo geométrico.

5.- La elaboración de un pre-test y post-test permitió observar la mejora significativa de los estudiantes, así como las deficiencias en el desarrollo de problemas de tasas de variación.

6.- Los estudiantes que participaron en el grupo experimental lograron a través de situaciones de visualización y modelado contextualizada la comprensión del concepto de la derivada desde una perspectiva más analítica e interpretativa.

7.- La enseñanza y aprendizaje utilizando estrategias didácticas apoyadas con GeoGebra, permite a los estudiantes investigar y mejorar su capacidad de abstracción, pensamiento lateral y persistencia mediante la integración de habilidades de pensamiento y comprensión conceptual.

8.- La experiencia con el diseño e implementación de esta estrategia didáctica muestra también la necesidad de crear nuevas situaciones en temas diversos, en las que se promueva de manera enfática la conversión del lenguaje natural al algebraico.

\section{REFERENCIAS}

Alvarado, O. A., Gallardo, H. J., y Vergel, M., Development of math skills in first semester students of public accounting, https://doi.org/10.1088/1742-6596/1408/1/012021, Journal of Physics: Conference Series, 1408, 1-4 (2019)

Arango, J., Gaviria, D., y Valencia, A., Differential Calculus Teaching through Virtual Learning Objects in the Field of Management Sciences, https://doi.org/10.1016/j.sbspro.2015.01.490, Procedia - Social and Behavioral Sciences, 176, 412-418 (2015)

Areaya, S., y Sidelil, A., Students' difficulties and misconceptions in learning concepts of limit, continuity and derivative, The Ethiopian Journal of Education, ISSN: 0425-4414, 32 (2), 1-38 (2012)

Báez, N., Pérez, O. L., y Blanco, R., Los registros de representación semiótica como vía de materialización de los postulados vigotskianos sobre pensamiento y lenguaje, https://doi.org/10.18359/ravi.2241, Academia y virtualidad, 11 (1), 16-26 (2018)

Berry, J. S., y Nyman, M. A., Promoting students' graphical understanding of the calculus, https://doi.org/10.1016/j.jmathb.2003.09.006, The Journal of Mathematical Behavior, 22 (4), $479-495$ (2003)

Caligaris, M., Schivo, M., y Romiti, M., Calculus y GeoGebra, an interesting partnership, https://doi.org/10.1016/j.sbspro.2015.01.735, Procedia-Social and Behavioral Sciences, 174, 1183-1188 (2015)

Cisneros, E. J., Jorquera, M. C., y Aguilar, A. M., Validación de instrumentos de evaluación docente en el contexto de una universidad española, https://doi.org/10.18175/vys3.1.2012.03, Voces y Silencios: Revista Latinoamericana de Educación, 3(1), 41-55 (2012)

Criscuolo, A., y Gnudi, A., Study of functions in a GeoGebra environment during "learning week" North American, GeoGebra Journal, ISSN: 2162-3856, 2(1), 24-30 (2013)

D'Zurilla, T. J., y Nezu, A. M., Problem-solving therapy: A positive approach to clinical intervention (3 $3^{\underline{a}}$ ed.). Nueva York: Springer (2007)

Duval, R., Representación, visión y visualización: Funciones cognitivas en el pensamiento matemático. Université du Littoral Côte-d'Opale, Boulogne, et Centre IUFM Nord Pas-de Calais, Lille (2000)

Duval, R., A cognitive analysis of problems of comprehension in a learning of mathematics. Journal of Educational Studies in Mathematic, 61(1-2), 103-131 (2006)

Feo, R., Orientaciones básicas para el diseño de estrategias didácticas, Tendencias pedagógicas, ISSN: 1989-8614, 16, 220-236 (2010)

Fortea, M., Experiencias e innovación de la docencia universitaria. España: Universitat Jaume (2003) 
Hartini, T. I., Liliasari, A. S., y Ramalis, T. R., Implementing Analytic Mechanics Learning Based on Multiple Representations on GeoGebra Software: In Forwardness to Face the Industrial Revolution 4.0 (Mr-Geo.4ir), Talent Development y Excellence, 12 (1), 3940-3954 (2020)

Kim, K.M., y Md-Ali, R., GeoGebra: Towards Realizing 21st Century Learning in Mathematics Education, Malaysian Journal of Learning and Instruction, Special Issues 2017, 93-115 (2017)

Krutetskii, V. A., The psychology of mathematical abilities in school children. University of Chicago Press, Chicago, USA: (1976)

Larson, R., Hostetler, R., y Edwards, B., Cálculo I (8va), Pirámide Ediciones S.A.: México (2006)

Md-Ali, R., y Kim, K. M., Geogebra in learning of mathematics towards supporting 'stem' education, https://doi.org/10.32861/jssr.spi6.776.782, Journal of Social Sciences Research, Special Issues 6, 776-782 (2018)

Molon, J. y Sidney, E., Cálculo no Ensino Médio: Uma abordagem possível e necessária com auxílio do Software GeoGebra, https://doi.org/10.5902/2179460X14523, Ciencia e Natura, 37, 156-178 (2015)

Montenegro, D. J., Interacción comunicativa con Blackboard Collaborate y el rendimiento académico de los estudiantes de educación a distancia, http://dx.doi.org/10.21503/hamu.v3i2.1322, Hamut'ay, 3 (2), 68-82 (2016)

Mora, C. D., Estrategias para el aprendizaje y la enseñanza de las matemáticas, Revista de Pedagogía, ISSN: 07989792, 24 (70), 181-272 (2003)

Olivares, J., y Valero, E., Resolving non-homogeneous linear differential equations using the undetermined method coefficients and variation of parameters by means of GeoGebra, http://dx.doi.org/10.1088/1742-6596/1391/1/012057, Journal of Physics: Conference Series, 1391, 1-5 (2019)

Orhun, N., Graphical understanding in mathematics education: Derivative functions and students' difficulties, https://doi.org/10.1016/j.sbspro.2012.09.551, Procedia-Social and Behavioral Sciences, 55, 679-684 (2012)

Ortiz, D., El constructivismo como teoría y método de enseñanza, https://doi.org/10.17163/soph.n19.2015.04, SoФia, Colección de Filosofía de la Educación, 19, 93-110 (2015)

Polya, G., Cómo plantear y resolver problemas. México: Trillas (1965)

Prodromou, T., GeoGebra in teaching and learning introductory statistics, https://doi.org/10.3991/ijet.v14i08.9581, The Turkish J. of Educ. Technol., 8(2), 53-67 (2015)

Rojas, J. P., Vergel, M., y Pabón, J. A., Understanding of derivative as an essential part of the study of differential calculus, https://doi.org/10.1088/1742-6596/1329/1/012014, Journal of Physics: Conference Series, 1329, 1-5 (2019)

Saldarriaga-Zambrano, P. J., Bravo-Cedeño, G., y Loor-Rivadeneira, M. R., La teoría constructivista de Jean Piaget y su significado para la pedagogía contemporánea, Revista Científica Dominio de las Ciencias, ISSN: 2477-8818, 2, 127-137 (2016)

Villalón, R., Luna. M., y García-Barrera, A., Valoración y uso de la plataforma Blackboard Collaborate en una universidad a distancia: estudio de caso sobre las prácticas declaradas de docentes del Grado de Psicología, Digital Education Review, ISSN: 2013-9144, 35, 267-288 (2019)

Widjajanti, K., Nusantara T., y otros cinco autores., Delaying scaffolding using GeoGebra: Improving the ability of vocational students to draw conclusions, https://doi.org/10.18421/TEM81-42, TEM Journal, 8(1), 305-310 (2019) 